\title{
Frontofacionasal dysplasia
}

INSERM

\section{Source}

INSERM. (1999). Orphanet: an online rare disease and orphan drug data base.

Frontofacionasal dysplasia. ORPHA:1791

Fronto-facio-nasal dysostosis is characterized by multiple craniofacial anomalies

(brachycephaly, blepharophimosis, ptosis, S-shaped palpebral fissures, coloboma, cleft lip and palate, deformed nostrils, encephalocele, hypertelorism, midface hypoplasia, malformed eyes, and absent inner eyelashes). 